\title{
A Faster Atomic Compass
}

\author{
An update to the polarization of the laser light used in an atom-based \\ compass allows the technology to reveal the 3D alignment of a magnetic \\ field in one snapshot rather than many.
}

By Christopher Crockett

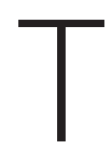
he traditional compass consists of a needle or compass rose that aligns itself to magnetic north. But there are other types of compasses. For example, the precession of atomic dipoles in a gas can also reveal a magnetic field's orientation. Such atomic compasses are useful for high-precision applications from medical diagnostics to quantum technologies, but existing approaches require watching the behavior of the atoms over time. Now, researchers have developed an atomic compass that reveals the 3D alignment of an external magnetic field in one snapshot [1].

When atoms interact with laser light, the shapes of their orbitals distort in response to the light's electromagnetic field. This distortion aligns the pointing direction of atoms' spins so that it matches that of the laser's polarization. Apply an external magnetic field to the atoms, and the spins precess, with spins perpendicular to the field wobbling in wider circles than those that are nearly parallel to it. The magnitude of the wobble alters the light-matter interaction, changing how much of the laser light the atoms absorb. By firing laser light at the atoms from

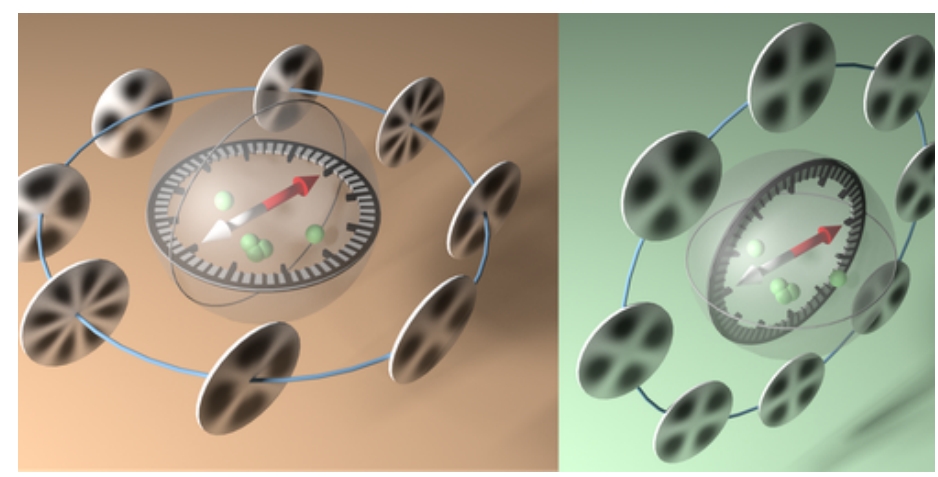

Credit: T. Clark/Hungarian Academy of Sciences multiple directions, researchers can deduce the magnetic field vector one component at a time.

Sonja Franke-Arnold of the University of Glasgow, UK, and colleagues instead used a vectorial laser-a laser where the polarization changes direction across the beam's profile. To demonstrate the technique, the team used their vectorial laser to illuminate a trapped cloud of rubidium atoms, to which they applied an external magnetic field. A camera captured the intensity of the light that exited the cloud. A Fourier analysis of the resulting spatial absorption pattern revealed the full 3D alignment of the applied magnetic field.

Christopher Crockett is a freelance writer based in Arlington, Virginia.

\section{REFERENCES}

1. F. Castellucci et al., "Atomic compass: Detecting 3D magnetic field alignment with vector vortex light," Phys. Rev. Lett. 127, 233202 (2021). 